\title{
Theme: Pediatric Neurology
}

\section{Is immunotherapy warranted for pediatric epilepsy patients with neuronal antibodies? (Epilepsia. 2016;57:823-31)}

Neuronal antibodies have been described in patients with newonset pediatric epilepsy without encephalitis. However, it remains ambiguous whether routine testing for neuronal antibodies and administration of immunotherapy among the seropositives would affect long-term outcome. In this study, stored blood samples were tested on cohort of children with epilepsy who did not receive any immunotherapy for neuronal antibodies. Seventeen patients $(9.5 \%)$ were positive for antibodies (3 against VGKC complex, 7 against NMDAR, 4 against CASPR2 and 3 against contactin-2). However, the titers were found to be relatively low $(\leq 1: 100$ for cell-surface antibodies). Moreover, it was found that response to standard antiepileptic drug and long term outcome did not differ from antibody-negative patients. Authors concluded that routine antibody testing in new-onset pediatric epilepsy is unlikely to be helpful.

Magnetic resonance spectroscopy for evaluation of children with seizures (Pediatr Neurol. 2016;58:57-66)

This study was designed to determine utility of addition of magnetic resonance spectroscopy (MRS) to magnetic resonance imaging (MRI) in evaluation of children with seizures. Medical records of patients who underwent both MRS and MRI were reviewed. In 100 of 233 cases (43\%), MRS contributed information additional to MRI. Among these 100 cases, 40 cases prompted further investigations like suspicion of inborn errors of metabolism, 25 cases contributed to diagnosis like differentiating dysplasia versus neoplasm, and 36 cases helped in prognosis (e.g. hypoxic ischemic brain injury). Additional 25 cases were abnormal on MRS when their MRI brain was normal. Authors concluded that MRS is useful adjunct to MRI for children undergoing imaging for seizures.

Bedside optic nerve sheath diameter assessment for Identification of increased intracranial pressure (Pediatr Neurol. 2016;54:35-8)

Diagnosis of idiopathic intracranial hypertension (IIH) often relies on opening pressure of cerebrospinal fluid on lumbar puncture. This study aimed to determine utility of bedside assessment of optic nerve sheath diameter in patients with IIH. Thirteen patients aged 12 to 18 years, scheduled for an elective lumbar puncture with the suspicion of idiopathic intracranial hypertension, were enrolled in the study. Optic nerve sheath diameter was measured by ultrasonography before performing a sedated lumbar puncture for measuring cerebrospinal fluid opening pressure. Abnormal measurements were predefined as optic nerve sheath diameter less than $4.5 \mathrm{~mm}$ and a cerebrospinal fluid opening pressure greater than $20 \mathrm{cmH}_{2} \mathrm{O}$. Optic nerve sheath diameter was able to predict or rule out elevated intracranial pressure in all ten patients who had raised ICP on lumbar puncture. Authors concluded that non-invasive assessment of the optic nerve sheath diameter could help to identify patients with elevated intracranial pressure in idiopathic intracranial hypertension.

Afray Comparative Genomic Hybridization in (JChild Neurol. 2016;31:691-9)

It is often believed that yield of array comparative genomic hybridization (CGH) increases with severity of intellectual disability. The present study aimed to determine association between positive Array CGH and clinical severity of intellectual disability (DeVries clinical score). Study population included 329 children with intellectual disability whose extensive investigations failed to reveal any etiological diagnosis. Microarray-based CGH identified pathogenic copy number variations (CNVs) in 52 cases $(16 \%)$, of whom $52 \%$ of patients showed DeVries score greater than 3. Causative CNVs were frequently found in cases of mild intellectual disability (30.8\%). Authors concluded that microarray-based CGH performs well on all individuals with intellectual disability/developmental delay, regardless of the severity.

Risk factors for subsequent febrile seizure (Epilepsia. 2016;57:1042-7)

This study aimed at identifying the risk factor for developing subsequent febrile seizure (FS) in children with a first febrile status epilepticus (FSE) compared to first simple febrile seizure (SFS). Among 294 enrolled children with first febrile seizure, 193 had FSE and rest 101 had FS. Risk of recurrence of second FS was $42.9 \%$ in FSE and $28.9 \%$ in FS $(P=0.09)$. However, risk of recurrence of FSE was $9.9 \%$ in FSE and $2.3 \%$ in SFS. Authors concluded that risk of subsequent FSE would be higher among those with previous FSE.

JAYA SHANKAR KAUSHIK jayashankarkaushik@gmail.com 